\title{
Serrated carcinoma arising from a sessile serrated adenoma
}

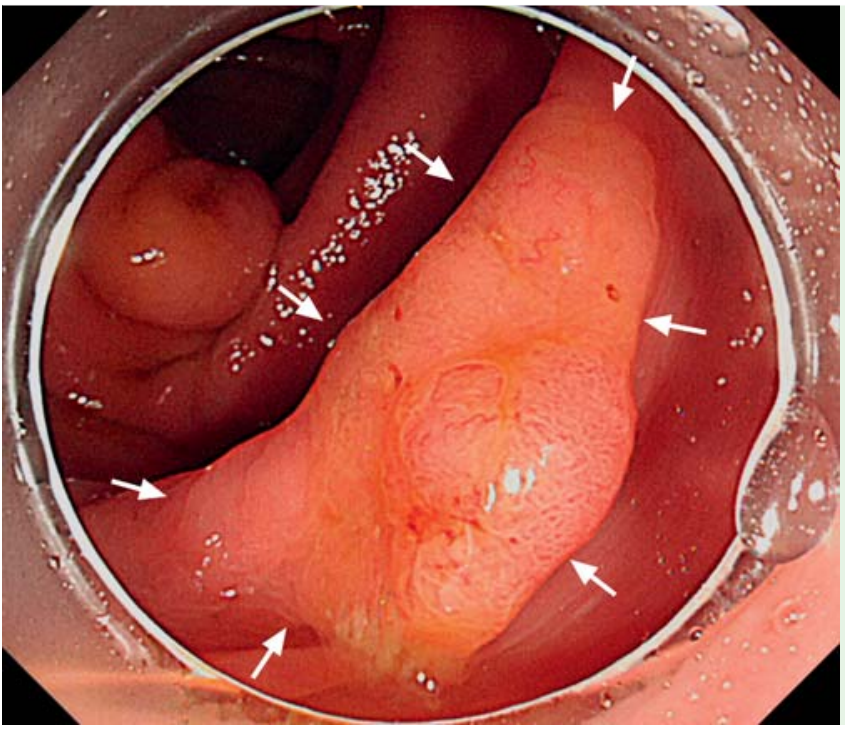

Fig. 1 Endoscopic image showing a 27-mm laterally spreading tumor in the ascending colon (arrows) with a mucuscovered surface that has a flat elevated appearance except for the peripheral portion, which displays a nodular surface.

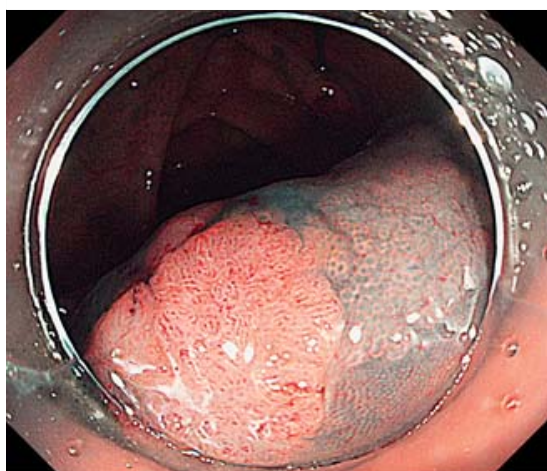

Fig. 2 Endoscopic appearance after submucosal injection showing a type III (tubular) pit pattern in the nodular area and a type II (stellate) pit pattern in the flat area.

Sessile serrated adenoma/polyp (SSA/P), a distinct serrated polyp subtype, can progress to colorectal cancer via the serrated neoplasia pathway [1]. On endoscopic examination, SSA/Ps usually show a flat or sessile appearance, a mucus covering, and a type II pit pattern. The endoscopic findings of uncomplicated SSA/Ps are well known; however, the endoscopic features of serrated carcinomas arising from SSA/Ps have not been fully described [2,3]. Herein, we report a case of serrated carcinoma arising from an SSA/P in a 66-year-old man who underwent endoscopic submucosal dissection (ESD) following colorectal cancer screening.
Colonoscopy revealed a 27-mm laterally spreading tumor in the ascending colon ( Fig. 1). Most of the mass showed a flat elevated surface with a mucus covering, whereas the peripheral portion displayed a nodular appearance. The central area showed a type II (stellate) pit pattern, whilst the peripheral area displayed a type III (tubular) pit pattern ( $\bullet$ Fig.2). Because there was a considerable risk of piecemeal resection, the tumor was resected by ESD instead of by endoscopic mucosal resection (EMR) [4].

On gross examination, the mass comprised two main parts ( $\mathrm{Fig} .3 \mathrm{a}$ ). Histopathological examination of these areas showed that the flat elevated area met the SSA/P criteria (green line), the nodular reddish area corresponded to an intramucosal adenocarcinoma (red line), whilst histologic transition was noted between

Despite increasing attention being paid to SSA/Ps, the endoscopic findings of SSA/Ps that are showing early neoplastic progression are not yet well known. We believe the present case clearly shows a biphasic endoscopic appearance of an SSA/P transitioning to a serrated carcinoma.

\section{Endoscopy_UCTN_Code_CCL_1AD_2AB}

\section{Competing interests: None} the two areas (orange line; $\bullet$ Fig. $\mathbf{3 b}, \mathbf{c}$ ).

\section{E.-J. Lee' ${ }^{1}$, M.-J. Kim², J. P. Suh ${ }^{3}$, S. H. Lee}

${ }^{1}$ Department of Surgery, Daehang Hospital, Seoul, Republic of Korea

2 Department of Pathology, Daehang Hospital, Seoul, Republic of Korea

${ }^{3}$ Department of Internal Medicine, Daehang Hospital, Seoul, Republic of Korea

\section{References}

1 Snover DC. Update on the serrated pathway to colorectal carcinoma. Hum Pathol 2011; 42: $1-10$

2 Huang CS, Farraye FA, Yang $S$ et al. The clinical significance of serrated polyps. Am J Gastroenterol 2011; 106: 229-240

3 Rex DK, Ahnen DJ, Baron JA et al. Serrated lesions of the colorectum: review and recommendations from an expert panel. Am J Gastroenterol 2012; 107: 1315-1329

4 Lee EJ, Lee JB, Lee SH et al. Endoscopic treatment of large colorectal tumors: comparison of endoscopic mucosal resection, endoscopic mucosal resection-precutting, and endoscopic submucosal dissection. Surg Endosc 2012; 26: 2220-2230

\section{Bibliography}

DOI http://dx.doi.org/

10.1055/s-0032-1326279

Endoscopy 2013; 45: E110-E111

(c) Georg Thieme Verlag KG

Stuttgart · New York

ISSN 0013-726X

\section{Corresponding author}

\section{E.J. Lee, MD}

Department of Surgery

Daehang Hospital

481-10 Bangbae Seocho

Seoul 137-820

Republic of Korea

Fax: +82-2-63888115

ejlee1009@naver.com

Fig. 3 a, b,c see following page. 


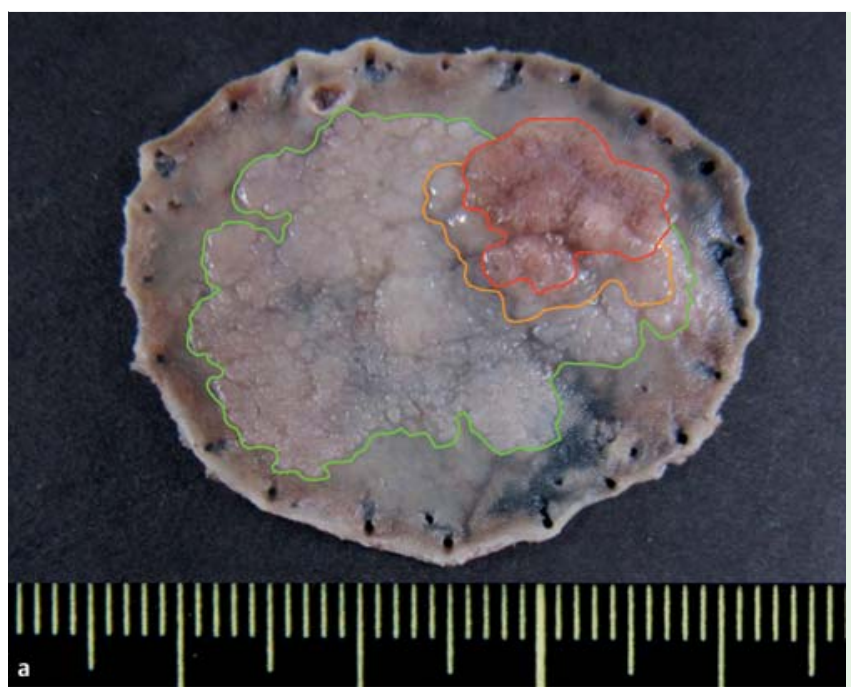

Fig.3 Appearances of the resected tumor.

a Gross appearance showing a biphasic tumor with a flat elevated area

(green line), nodular area (red line), and an intervening transitional area (orange line).

b Low power view of the tumor with the three areas indicated by their respective colors on the bar below (original magnification $\times 12.5$ ). c High power view of each of the tumor areas showing features consistent with an SSA/P in the green area, an intramucosal adenocarcinoma in the red area and an area with low grade dysplasia in the transitional orange area (original magnification $\times 200$ ).

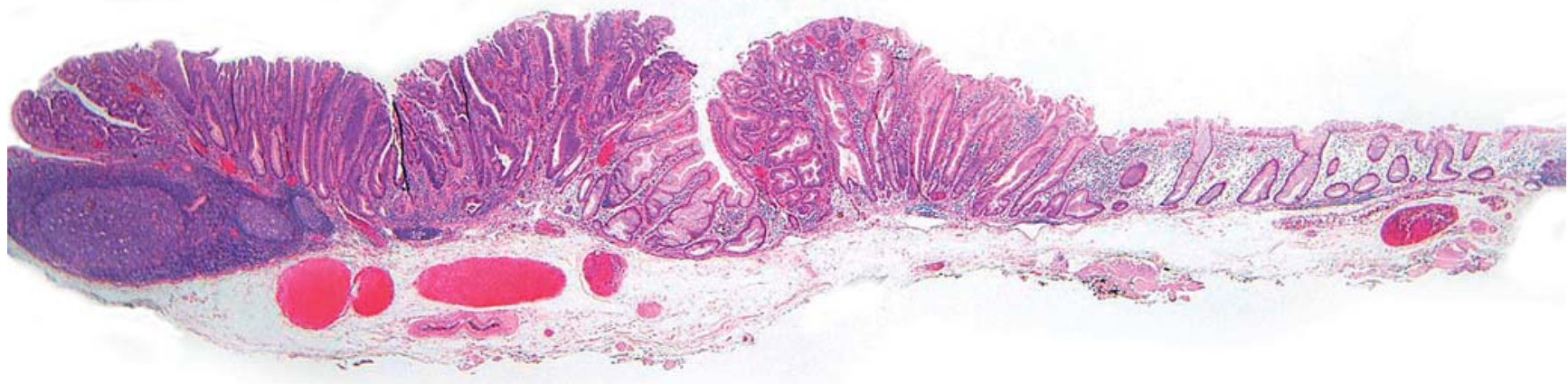

b
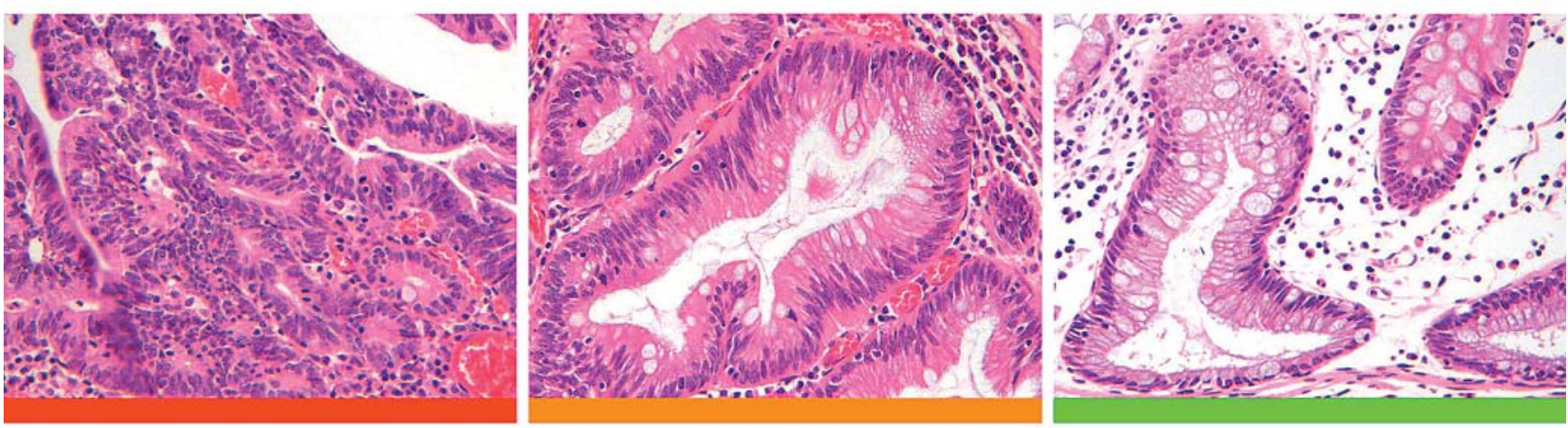